\title{
Monomeric C-reactive protein level is associated with osteoarthritis
}

\author{
YULIN LIANG $^{1 *}, \mathrm{KE} \mathrm{XU}^{2 *}$, WENGUANG LIU ${ }^{2}$, XIAOLING LIU ${ }^{1}$, \\ PING YUAN ${ }^{1}$, PENG XU ${ }^{2}$ and HAIYUN $\mathrm{LI}^{3}$
}

\begin{abstract}
${ }^{1}$ Key Laboratory of Cell Activities and Stress Adaptations, Ministry of Education, School of Life Sciences, Lanzhou University, Lanzhou, Gansu 730000; ${ }^{2}$ Department of Joint Surgery, Hong Hui Hospital, Xi'an Jiaotong University, Xi'an, Shaanxi 710054; ${ }^{3} \mathrm{MOE}$ Key Laboratory of Environment and Genes Related to Diseases, Ministry of Education, School of Basic Medical Sciences, Xi'an Jiaotong University, Xi'an, Shaanxi 710061, P.R. China
\end{abstract}

Received December 10, 2021; Accepted January 21, 2022

DOI: $10.3892 /$ etm.2022.11206

\begin{abstract}
Osteoarthritis (OA) is a chronic joint disease characterized by articular cartilage degeneration and secondary bone hyperplasia. $\mathrm{C}$-reactive protein (CRP) is an acute-phase protein that is widely used as a marker of inflammation. Elevated plasma levels of CRP are commonly observed in patients with OA during the acute phase. Current evidence indicates that CRP dissociating into a monomeric form (mCRP) is the main functional conformation at inflammatory loci. However, it remains unclear whether mCRP is associated with OA and whether mCRP can be used as a biomarker for its pathogenesis. In the present study, the concentration of CRP, mCRP and anti-mCRP autoantibody were detected by performing ELISA. The levels of plasma CRP, mCRP and anti-mCRP autoantibody between healthy subjects and patients with OA were compared. The results revealed that plasma mCRP was strongly associated with OA, while mCRP autoantibodies exhibited little correlation with this condition. Additionally, it was identified that the plasma mCRP levels in Kellgren-Lawrence (KL) grade 4 patients were significantly higher than in those with KL grade 3 . Thus, it was revealed in the present study that plasma level of mCRP is associated with OA, which may directly reflect the disease degree of patients.
\end{abstract}

Correspondence to: Professor Peng $\mathrm{Xu}$, Department of Joint Surgery, Hong Hui Hospital, Xi'an Jiaotong University, 555 Youyi East Road, Xi'an, Shaanxi 710054, P.R. China

E-mail: sousou369@163.com

Professor Haiyun Li, MOE Key Laboratory of Environment and Genes Related to Diseases, Ministry of Education, School of Basic Medical Sciences, Xi'an Jiaotong University, 76 Yanta West Road, Xi'an, Shaanxi 710061, P.R. China

E-mail: lihaiy@xjtu.edu.cn

*Contributed equally

Key words: osteoarthritis, monomeric C-reactive protein, inflammation, Kellgren-Lawrence grade
Therefore, mCRP may be a potential indicator that can be used to monitor the disease activity and evaluate the efficiency of OA therapy.

\section{Introduction}

Osteoarthritis (OA) is also known as degenerative arthropathy and proliferative OA. The different names are derived from the pathological manifestations of arthropathy, which include both cartilage degeneration and the formation of new bone $(1,2)$. The clinical manifestations of this disease include joint swelling, pain, joint effusion and hyper-osteogeny (3). The incidence of OA increases with age and it is a common joint disease in the elderly population (4). At present, the pathogenesis of OA has not been determined. Previous studies have demonstrated that inflammatory responses are involved in the pathogenesis of $\mathrm{OA}$ and that pathological changes in the synovial structure are similar to that of rheumatoid arthritis at the early stages of onset (5-7). At present, OA is diagnosed mainly through the combination of symptoms and signs with imaging examination. Therefore, the early diagnosis of OA, differentiation of the severity and the search for appropriate markers have become important topics in clinical research.

C-reactive protein (CRP), a member of the pentraxin family, is composed of five identical subunits linked by non-covalent bonds (8). CRP is widely found in vertebrates and invertebrates, and its structure and sequence demonstrate high evolutionary conservation, suggesting that this protein has important biological significance (9). CRP is an acute phase protein whose plasma concentration rises rapidly when the body is affected by tissue damage or bacterial infection (10-13). It has been widely used in clinical practice for the diagnosis and detection of various infectious and autoimmune diseases $(11,14)$. CRP not only serves as an inflammatory marker but also serves numerous important physiological functions in innate immune responses, such as activating complement pathways or regulating macrophage phagocytosis through $F c \gamma$ receptors to help clear cell debris and apoptotic cells (15-17). A previous study indicated that monomeric CRP (mCRP) is an allosteric active form of CRP involved in the inflammatory process, while pentameric CRP may serve as a 
protein library capable of transforming into mCRP (18). In the present study, the clinical samples of patients with OA were collected and the differences and associations between CRP, $\mathrm{mCRP}$ and anti-mCRP autoantibodies in the serum of healthy subjects and patients were analyzed, with the aim of providing evidence for the pathogenesis of OA and the use of mCRP as a novel clinical marker.

\section{Materials and methods}

Materials and reagents. Human CRP (cat. no. BP300.X; lot nos. 361639 and 404353) purified from ascites was purchased from the Binding Site. mCRP was prepared by treating CRP with $8 \mathrm{M}$ urea-EDTA (cat. no. JT8991-1; lot no. 0000221293; Avantor, Inc.) (19) or by recombinant expression and purification as previously described $(20,21)$. Sheep anti-human CRP polyclonal antibody (cat. no. PC044; lot nos. 352325 and 076682) was obtained from the Binding Site. Mouse anti-human CRP monoclonal antibody (mAb) CRP-8 (cat. no. C1688; lot no. 025M4863V) was obtained from Sigma-Aldrich; Merck KGaA. Mouse anti-human CRP mAb 1D6 was generated as previously described $(22,23)$. HRP-labeled goat anti-mouse IgG (cat. no. 115-035-003; lot no. 125229) was purchased from Jackson ImmunoResearch Laboratories, Inc. HRP-labeled donkey anti-sheep IgG (cat. no. ab6900; lot no. GR272029-6) and HRP-labeled mouse anti-human IgG (cat. no. ab99757) were both obtained from Abcam.

Patient selection. A total of 206 patients with OA diagnosed between January 2016 and July 2018 at the Department of Joint Surgery and Joint Reconstruction of Xi'an Hong Hui Hospital (Xi'an, China) were enrolled in the present study. Participants were assessed by rheumatologists and radiologists. Rheumatoid arthritis or any other form of inflammatory arthritis (such as crystal arthropathy or septic arthritis) were considered to be exclusion criteria. A total of 60 age and sex matched healthy individuals were selected from the same hospital between November 2017 and July 2018. The clinicopathological characteristics of patients with OA are listed in Table I. CRP, mCRP and anti-mCRP autoantibodies were detected by ELISA while other clinical features were collected hospital case records. The demographic data of OA patients and healthy individuals are included in Table II. The plasma samples of patients were obtained prior to the initiation of medical treatment. All samples were stored at $-80^{\circ} \mathrm{C}$ in aliquots. Informed consent for blood sample collection was signed by all participants. The present study followed the guidelines of The Declaration of Helsinki and was approved (approval no. 202003055) by the Ethics Committee of the Hong Hui Hospital (Xi'an, China).

Patient classification. The Kellgren-Lawrence (KL) grading system of knee OA is the grading method of knee OA severity. According to the X-ray manifestations of the knee joint, OA severity was divided into grade 0 (normal knee joint), grade 1,2,3 and 4 (the most serious knee OA) $(24,25)$ and were described as follows: i) Grade 0: Knee joint X-ray is completely normal. There are no manifestations of OA. No joint space stenosis and no reactive bone changes can be observed. ii) Grade 1: Suspected knee joint space stenosis.
Osteophytes may occur, but only slightly. iii) Grade 2: Small osteophytes and possible joint space narrowing are identifiable on the X-ray of a standing knee joint. iv) Grade 3: KL grade 3 of knee OA is characterized by a large number of moderate osteophytes, clear narrowing of joint space, certain subchondral bone sclerosis (increased white area with joint edges is identifiable via X-ray) and possible knee deformity. v) Grade 4: KL grade 4 of knee OA is characterized by a large number of large osteophytes, severe narrowing of joint space, obvious subchondral bone sclerosis and obvious knee deformity. The patients with OA mentioned in the present study were all patients who received systematic evaluation and treatment at hospital. In the early stages of OA, patients have occasional pain in the knee joint, which affects exercise but rarely daily life. If patients pay attention to rest and exercise properly, they can alleviate their symptoms $(26,27)$. For the aforementioned reasons, there are no hospitalized patients in the early stage of KL grade 1 and KL grade 2. Therefore, patients with KL grade 1 and $K L$ grade 2 were not included in the present study. KL grade 3 and KL grade 4 patients in the middle and late stage exhibit joint degeneration and pain aggravation, which seriously affects their daily life, requiring hospitalization or joint replacement $(26,27)$. These patients were the main research subjects in the present study.

ELISA assay quantifying CRP. The sheep anti-human CRP polyclonal antibody was immobilized onto microtiter wells (1:2,000; cat. no. 42592; lot no. 10917007; Corning, Inc.) in coating buffer (10 mM sodium carbonate/bicarbonate, $\mathrm{pH} 9.6)$ overnight at $4^{\circ} \mathrm{C}$. All the following steps were conducted at $37^{\circ} \mathrm{C}$, and after each incubation step, wells were washed three times with TBS (10 mM Tris, $140 \mathrm{mM} \mathrm{NaCl}, \mathrm{pH}$ 7.4) containing $0.02 \%$ NP-40. Wells were blocked with blocking buffer [TBS containing $1 \%$ bovine serum albumin (cat. no. 0322; LABLEAD)] for $1 \mathrm{~h}$. Samples diluted in blocking buffer were added into wells for $1 \mathrm{~h}$. Captured CRP was detected with 1D6 $\mathrm{mAb}$ (1:100 in blocking buffer) that specifically recognizes its native conformation and an HRP-labeled goat anti-mouse IgG (1:20,000 in blocking buffer). Wells were incubated with TMB buffer [0.1 mg/ml 3,3,5,5-tetramethylbenzidine (cat. no. 0759; LABLEAD) and $0.02 \% \mathrm{H}_{2} \mathrm{O}_{2}$ in $0.1 \mathrm{M} \mathrm{Na}_{2} \mathrm{HPO}_{4}, 0.05 \mathrm{M}$ citric acid, $\mathrm{pH}$ 4.5-5.5] for $30 \mathrm{~min}$ and stopped with $1 \mathrm{M} \mathrm{H}_{2} \mathrm{SO}_{4}$. The optical density (OD) of samples were measured at 570 and $450 \mathrm{~nm}$ using a microplate reader. The OD value of each sample was calculated as OD450-OD570 nm. A total of $100 \mu \mathrm{l}$ volume was used at all incubation steps, while $300 \mu \mathrm{l}$ volume was used for washing after each incubation step.

ELISA assay quantifying $m C R P$. Mouse anti-human CRP $\mathrm{mAb}$ CRP-8 was immobilized onto microtiter wells at $0.3 \mu \mathrm{l} / \mathrm{ml}$ in coating buffer overnight at $4^{\circ} \mathrm{C}$. All the following steps were conducted at $37^{\circ} \mathrm{C}$, and after each incubation step, wells were washed three times with TBS containing $0.02 \%$ NP-40. Wells were blocked with blocking buffer for $1 \mathrm{~h}$. Samples diluted in blocking buffer were added into wells for $1 \mathrm{~h}$. Captured mCRP was detected with a sheep anti-human CRP polyclonal antibody (1:2,000 in blocking buffer) which can both recognize the CRP, mCRP and an HRP-labeled donkey anti-sheep IgG (1:20,000 in blocking buffer). Wells were incubated with TMB buffer for $30 \mathrm{~min}$ and stopped with $1 \mathrm{M} \mathrm{H}_{2} \mathrm{SO}_{4}$. Absorbance 
Table I. Clinical characteristics of patients with osteoarthritis.

Value (total number, mean \pm SD or median)

Clinical characteristics

All OA patients

OA patients that were KL graded

Sex

Female

Male

Age, years

Grade

KL3

KL4

$\mathrm{CRP}(\mu \mathrm{g} / \mathrm{ml})$

$\mathrm{mCRP}(\mathrm{ng} / \mathrm{ml})$

Anti-mCRP autoantibody (OD)

Neutrophil ratio (\%)

Lymphocyte ratio (\%)

Monocyte ratio (\%)

Uric acid $(\mu \mathrm{mol} / \mathrm{l})$

Urea (mmol/l)

Creatinine $(\mu \mathrm{mol} / \mathrm{l})$

Total cholesterol (mmol/l)

Triglyceride (mmol/l)

High density lipoprotein ( $\mathrm{mmol} / \mathrm{l})$

Low density lipoprotein ( $\mathrm{mmol} / \mathrm{l})$

Apolipoprotein A1 (g/l)

Apolipoprotein B (g/l)

Glucose (mmol/l)

Antistreptococcolysin O (IU/ml)

Rheumatoid factor (IU/ml)
159

47

$66.55 \pm 8.16$

14

35

$0.77(0.37-1.66)$

$12.51(7.78-24.81)$

$0.52 \pm 0.37$

$73.51 \pm 8.49$

$17.1 \pm 6.16$

$7.1(5.8-8.3)$

$293.06 \pm 70.1$

$5.5(4.5-6.6)$

$58(50-66.25)$

$4.46 \pm 0.94$

1.38 (1.03-1.84)

$1.23(1.09-1.39)$

$2.85(2.33-3.3)$

$1.35 \pm 0.24$

$0.88(0.75-1.04)$

4.88 (4.44-5.42)

34.5 (18-59)

$7.2(3.25-8.9)$
37

12

$68 \pm 8.28$

14

35

$2.17(0.61-4.97)$

15.95 (8.45-24.1)

$0.46 \pm 0.27$

$74.16 \pm 6.86$

$16.71 \pm 6.15$

7.05 (6.18-8.23)

$301.63 \pm 75.63$

5.7 (4.57-6.65)

57 (49.75-66.5)

$4.47 \pm 0.97$

$1.4(1.1-1.76)$

$1.26(1.13-1.4)$

2.77 (2.35-3.29)

$1.37 \pm 0.22$

$0.84(0.75-1)$

$4.87(4.41-5.81)$

$32(16.5-60.5)$

$7.3(3.17-9.05)$

CRP, C-reactive protein; KL, Kellgren-Lawrence; mCRP, monomeric C-reactive protein.

Table II. Comparisons of patients with OA and healthy individuals.

\begin{tabular}{|c|c|c|c|}
\hline \multirow[b]{2}{*}{ Clinical characteristic } & \multicolumn{2}{|c|}{ Value (total number or mean $\pm \mathrm{SD}$ or median) } & \multirow[b]{2}{*}{ P-value } \\
\hline & $\mathrm{OA}$ & Healthy & \\
\hline Sex & & & - \\
\hline Female & 159 & 46 & \\
\hline Male & 47 & 14 & \\
\hline Age, years & $66.55 \pm 8.16$ & $65.35 \pm 5.35$ & 0.241447 \\
\hline $\mathrm{CRP}(\mu \mathrm{g} / \mathrm{ml})$ & $0.77(0.37-1.66)$ & $0.3(0.04-0.72)$ & $3.83 \times 10^{-5}$ \\
\hline $\mathrm{mCRP}(\mathrm{ng} / \mathrm{ml})$ & $12.51(7.78-24.81)$ & $5.04(3.45-9.77)$ & $3.72 \times 10^{-5}$ \\
\hline Anti-mCRP Autoantibody (OD) & $0.52 \pm 0.37$ & $0.46 \pm 0.24$ & 0.399173 \\
\hline
\end{tabular}

OA, osteoarthritis; CRP, C-reactive protein; mCRP, monomeric CRP.

at OD570 and OD450 $\mathrm{nm}$ was measured with a microplate reader. The $\mathrm{OD}$ value of each sample was calculated as OD450-OD570 $\mathrm{nm}$. A total of $100 \mu \mathrm{l}$ volume was used at all incubation steps, while $300 \mu \mathrm{l}$ volume was used for washing after each incubation step.
ELISA assay quantifying anti-mCRP autoantibody. The mCRP was immobilized onto microtiter wells at $2 \mu \mathrm{g} / \mathrm{ml}$ in coating buffer overnight at $4^{\circ} \mathrm{C}$. All the following steps were conducted at $37^{\circ} \mathrm{C}$, and after each incubation step wells were washed 3 times with TBS containing $0.02 \%$ 



Figure 1. Difference and correlation analysis of serum CRP, mCRP and anti-mCRP autoantibody. The plasma concentrations of (A) CRP, (B) mCRP and (C) anti-mCRP autoantibody in patients with OA were compared with healthy controls. The correlation of (D) CRP with mCRP, (E) mCRP with anti-mCRP autoantibody and (F) CRP with anti-mCRP autoantibody were analyzed using Pearson's correlation test. ${ }^{* * * *} \mathrm{P}<0.0001$ as indicated. CRP, C-reactive protein; mCRP, monomeric CRP; OA, osteoarthritis; OD, optical density; R, Pearson's correlation coefficient; ns, not significant.

NP-40. Wells were blocked with blocking buffer for $1 \mathrm{~h}$. Samples diluted in blocking buffer were added into wells for $1 \mathrm{~h}$. Captured anti-mCRP autoantibody was detected with an HRP-labeled mouse anti-human $\operatorname{IgG}(1: 20,000$ in blocking buffer). Wells were incubated with TMB buffer for $30 \mathrm{~min}$ and stopped with $1 \mathrm{M} \mathrm{H}_{2} \mathrm{SO}_{4}$. Absorbance at OD570 and OD450 $\mathrm{nm}$ was measured with a microplate reader. The OD value of each sample was calculated as OD450-OD570 nm. A total of $100 \mu \mathrm{l}$ volume was used at all incubation steps, while $300 \mu 1$ volume was used for washing after each incubation step.

Statistical analysis. Each sample was analyzed three times. The mean value of each sample was used for subsequent analysis. Differences between groups were all analyzed using the Mann-Whitney U-test. Correlations were all analyzed using the Pearson's correlation test. The Univariable logistic regression was used to determine contributors to $\mathrm{KL}$ grades in patients with OA. All analyses were performed with R 4.0.3 ( $\mathrm{R}$ Core Team) (28). $\mathrm{P}<0.05$ was considered to indicate a statistically significant difference.

\section{Results}

Difference and correlation analysis of serum CRP, $m C R P$ and anti-mCRP autoantibody. The demographic and clinical data of patients are summarized in Table I and continuous characteristics are presented as the mean $\pm \mathrm{SD}$ or median (interquartile range). Overall comparisons of patients with $\mathrm{OA}$ and healthy individuals are summarized in Table II. The differences in plasma levels of CRP, mCRP and anti-mCRP autoantibody between healthy subjects and patients with OA are revealed in Fig. 1. The level of plasma CRP in patients with OA was significantly higher than that in healthy individuals (Fig. 1A), as well as the

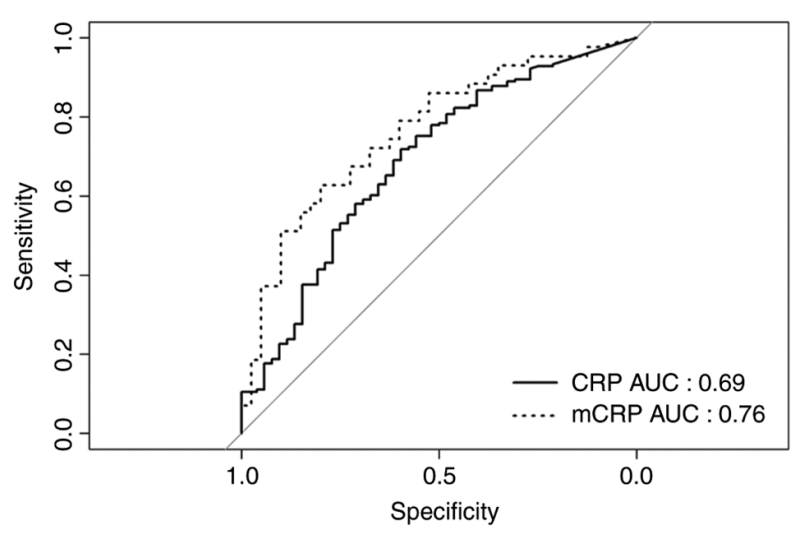

Figure 2. Receiver operating characteristic curves of CRP and mCRP for diagnosing osteoarthritis. mCRP (dotted line) has improved predictive performance than $\mathrm{CRP}$ (solid line). CRP, C-reactive protein; mCRP, monomeric CRP; AUC, area under the curve.

level of plasma mCRP (Fig. 1B). No significant difference in anti-mCRP autoantibody between patients with OA and healthy subjects was identified (Fig. 1C). The relationships among CRP, mCRP and anti-mCRP autoantibody are also illustrated in Fig. 1. CRP was strongly correlated with mCRP (Fig. 1D), probably due to mCRP being produced by the depolymerization of pentamer CRP at the inflammatory loci in the lesion area. mCRP was correlated with anti-mCRP autoantibody (Fig. 1E) most likely due to the fact that anti-mCRP autoantibody is generated by mCRP stimulation. No correlation was found between CRP and anti-mCRP autoantibody (Fig. 1F), which can be explained by CRP and anti-mCRP autoantibody not being directly related. In order to further investigate the correlation of CRP and $\mathrm{mCRP}$ with the risk of OA, receiver operating characteristic (ROC) curve analyses was performed (Fig. 2). The 
A

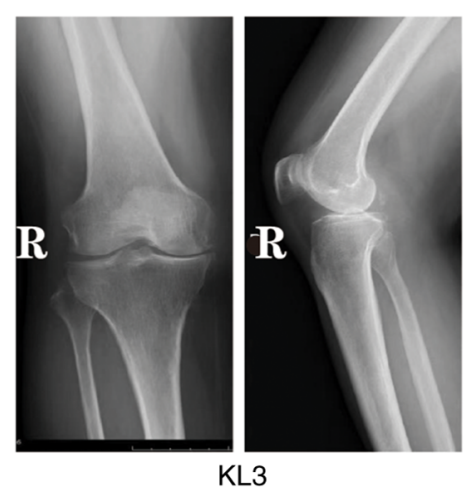

C



$E$



$\mathrm{B}$

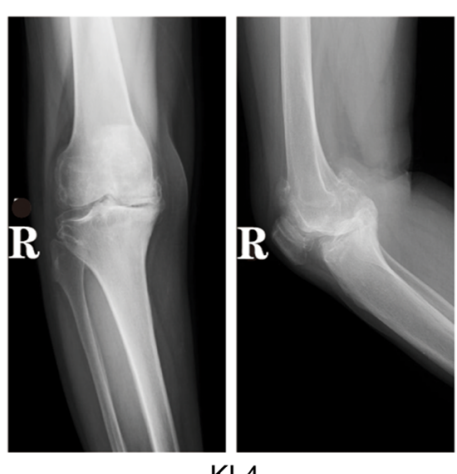

KL4

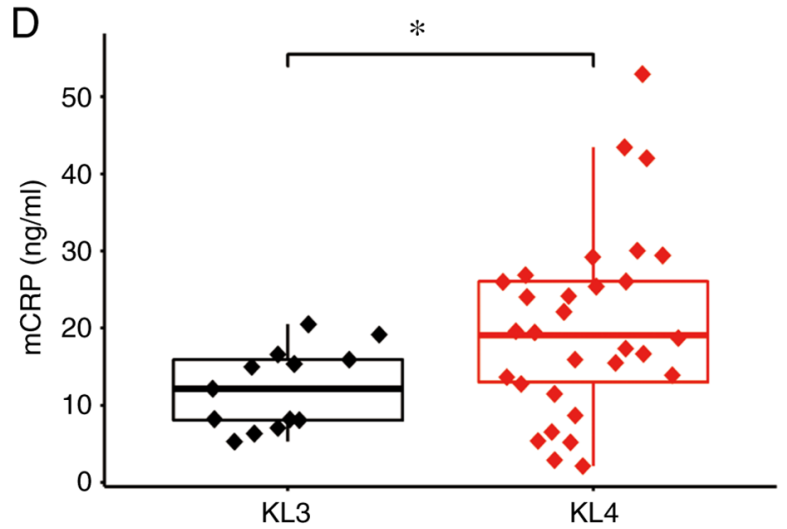

$\mathrm{F}$



Figure 3. Differences in CRP, mCRP, anti-mCRP autoantibody and the clinical features between KL grades in patients. Typical diagnostic images of (A) KL3 and (B) KL4 grades in patients with OA. Plasma concentrations of (C) CRP, (D) mCRP, (E) anti-mCRP autoantibody and (F) creatinine in patients with OA with KL grades. " $\mathrm{P}<0.05$ as indicated. CRP, C-reactive protein; mCRP, monomeric CRP; KL, Kellgren-Lawrence; OA, osteoarthritis; OD, optical density; $\mathrm{R}$, right-hand side; ns, not significant.

area under the CRP curve was 0.69 , and the area under the mCRP curve was 0.76 , which demonstrated that mCRP has improved predictive performance.

Differences in CRP, $m C R P$, anti-mCRP autoantibody and clinical features between $K L$ grades in patients with $O A$. Images of different KL grades are presented in Fig. 3A and B. Compared with those with KL grade 3, patients with KL grade 4 had a narrower articular cavity and more evident joint deformities. CRP and mCRP were revealed to be significantly higher in KL grade 4 than in KL grade 3 (Fig. 3C and D). These findings suggested that CRP and mCRP can be used as indicators of disease severity. There was no significant difference in anti-mCRP autoantibody between KL grades (Fig. 3E), suggesting that anti-mCRP autoantibody is not associated with the severity of OA. A significant difference in creatinine level between KL grades was also observed (Fig. 3F), while all other clinical features are not significantly different (data not shown). Univariate logistic regression was performed (Table III) and the results confirmed that CRP and mCRP are significantly associated with KL grades. This finding suggested that CRP and mCRP may be contributors to OA pathogenesis.

Associations of CRP, mCRP and anti-mCRP autoantibody with age and sex in patients. As it can be observed in Fig. 4, the differences in CRP, mCRP and anti-mCRP autoantibody between sex are all not significant (Fig. 4A-C). Thus, the patients were not grouped according to sex for further 
Table III. Univariate analysis of Kellgren-Lawrence grades of patients with osteoarthritis.

\begin{tabular}{|c|c|c|c|c|}
\hline Clinical feature & P-value & OR & CI95 lower limit & CI95 upper limit \\
\hline Sex & 0.753 & 1.269 & 0.307 & 6.534 \\
\hline Age & 0.161 & 1.057 & 0.980 & 1.148 \\
\hline CRP & 0.018 & 2.342 & 1.312 & 5.386 \\
\hline $\mathrm{mCRP}$ & 0.021 & 1.034 & 1.009 & 1.069 \\
\hline Autoantibody & 0.227 & 0.206 & 0.013 & 2.488 \\
\hline Neutrophils Ratio & 0.295 & 1.051 & 0.958 & 1.156 \\
\hline Lymphocytes Ratio & 0.227 & 0.939 & 0.843 & 1.039 \\
\hline Monocytes Ratio & 0.845 & 1.030 & 0.773 & 1.421 \\
\hline Uric Acid & 0.447 & 1.003 & 0.995 & 1.013 \\
\hline Urea & 0.326 & 1.248 & 0.825 & 2.029 \\
\hline Creatinine & 0.077 & 1.049 & 1.002 & 1.115 \\
\hline Total Cholesterol & 0.318 & 1.422 & 0.732 & 3.007 \\
\hline Triglyceride & 0.822 & 0.923 & 0.466 & 1.972 \\
\hline High Density Lipoprotein & 0.569 & 2.321 & 0.871 & 49.317 \\
\hline Low Density Lipoprotein & 0.431 & 1.369 & 0.656 & 3.250 \\
\hline Apolipoprotein A1 & 0.602 & 2.215 & 0.119 & 54.015 \\
\hline Apolipoprotein B & 0.536 & 2.480 & 0.161 & 58.439 \\
\hline Glucose & 0.523 & 0.844 & 0.488 & 1.466 \\
\hline Antistreptococcolysin $\mathrm{O}$ & 0.720 & 0.997 & 0.983 & 1.013 \\
\hline Rheumatoid Factor & 0.438 & 1.022 & 0.993 & 1.127 \\
\hline
\end{tabular}

CRP, C-reactive protein; mCRP, monomeric CRP; OR, odds ratio.
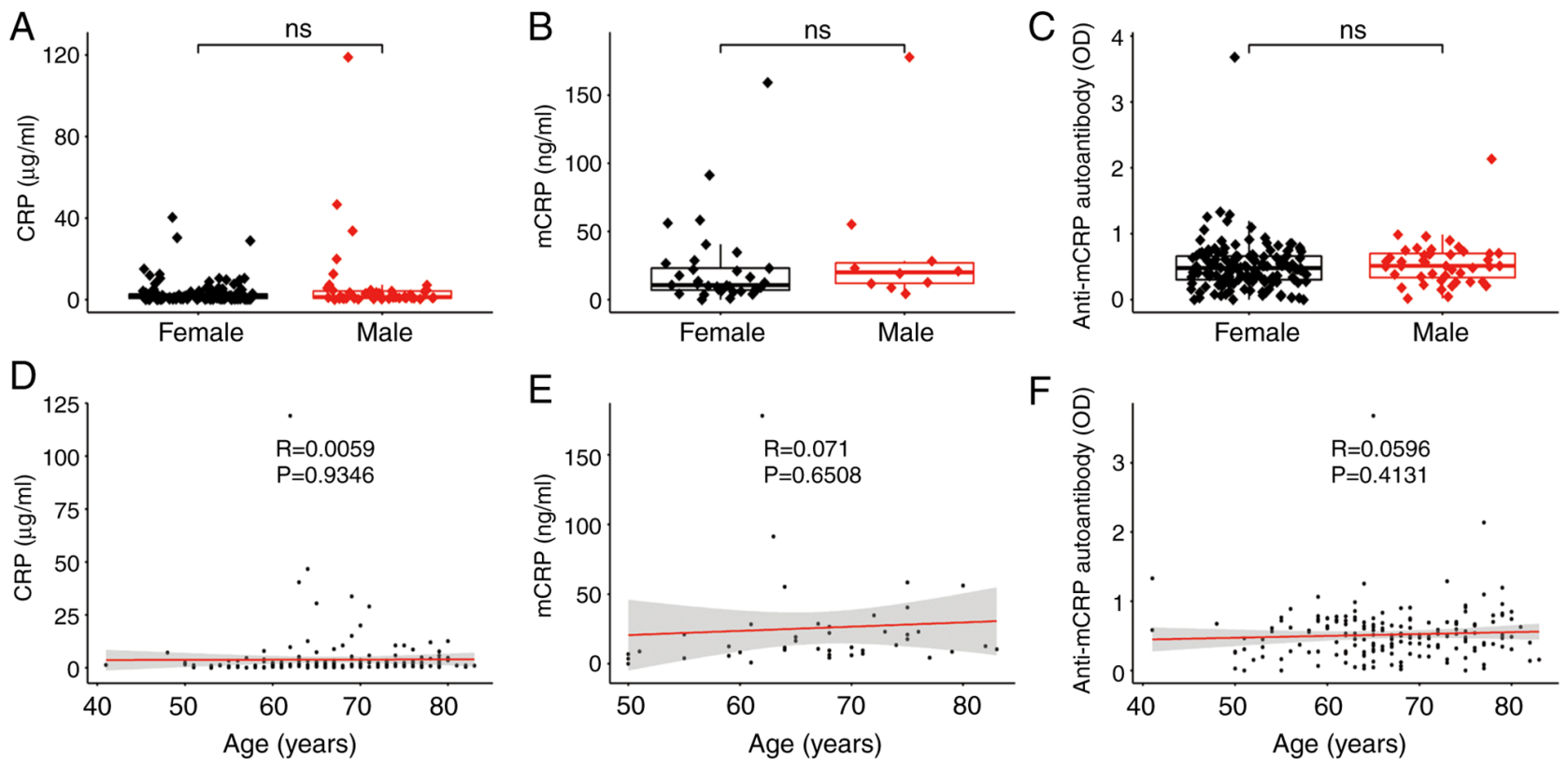

Figure 4. Association of CRP, mCRP and anti-mCRP autoantibody with the age and sex of patients with OA. Plasma concentrations of (A) CRP, (B) mCRP and (C) anti-mCRP autoantibody in male and female patients with OA. The correlations of age with (D) CRP, (E) mCRP (F) and anti-mCRP autoantibody were analyzed using Pearson's correlation test. CRP, C-reactive protein; mCRP, monomeric CRP; OA, osteoarthritis; R, Pearson's correlation coefficient; ns, not significant; OD, optical density.

subgroup analysis. Since the correlation of CRP, mCRP and anti-mCRP autoantibody with age was also not significant (Fig. 4D-F), the patients were again not grouped according to their age.

\section{Discussion}

According to a survey performed by the World Health Organization, the incidence of OA has reached $10 \%$ in people 
aged $>60$ years (29). At present, the diagnosis of OA mainly depends on imaging methods. Therefore, the development of convenient early methods of diagnosis for $\mathrm{OA}$ is a direction worthy of study.

CRP is an important marker of the inflammatory response, which increases rapidly in the acute stage of infection and tissue injury (10-13,30). Studies have also revealed that CRP was directly involved in the development of certain diseases. In myocardial infarction, for example, CRP mediates tissue damage mainly by activating complement pathways $(31,32)$. In addition, $\mathrm{mCRP}$ is the monomeric form of CRP and the main active conformation of local tissues (33-35). Furthermore, anti-mCRP autoantibody is generated by mCRP stimulation. Previous studies have determined that anti-mCRP autoantibody is associated with the degree of injury and prognosis of patients $(36,37)$. To study the relationship between CRP, mCRP, anti-mCRP autoantibody and OA, CRP, mCRP and anti-mCRP autoantibody were selected as observation objects to detect the difference and correlation between patients and healthy controls. Significant differences in plasma CRP and mCRP levels between patients and healthy controls and KL grades were identified, suggesting that they may be involved in the development of OA and could be used as severity markers of OA. There was a difference between CRP and mCRP, due to CRP being a non-specific inflammatory marker protein secreted by the liver and has a high concentration in plasma; $\mathrm{mCRP}$ is considered to play a role by depolymerizing pentamer CRP into monomer form in the local tissue of the lesion, and in addition, most of mCRP still resident in the local tissue $(18,38)$, so the concentration of mCRP in blood is low. In clinical application, it is easier to detect CRP with higher concentration by ELISA; However, compared with CRP, mCRP may be a more sensitive indicator in a specific disease. Combined with the ROC curve, mCRP has higher accuracy and prediction sensitivity.

The mCRP is the monomer form of pentamer CRP and inflammatory conditions in tissues can promote the dissociation of CRP to mCRP (39). In the process of dissociating to mCRP, CRP can expose certain new antigen epitopes, giving mCRP biological activities different from the pentameric protein. Previous studies have demonstrated that $\mathrm{mCRP}$ has a stronger biological effect. For example, mCRP, the main isomer form of CRP, can bind with natural and modified low density lipoprotein (40) and regulate complement activation more effectively (41). Furthermore, mCRP has a stronger activation effect on endothelial cells (7) and neutrophils (42), which means that mCRP formed by the structural rearrangement of CRP is a favorable complement of CRP function. These findings suggested that mCRP is the dominant form of CRP, functioning under pathological and physiological conditions. The present experimental results delineated that there was a strong correlation between serum $\mathrm{mCRP}$ and CRP in $\mathrm{OA}$ and that there were significant differences in $\mathrm{mCRP}$ between patients and healthy controls, as well as in different stages of OA, suggesting that CRP in OA may be involved in the development of the disease through dissociation into mCRP. The mostly commonly investigated pathways associated with OA are the Wnt, Notch, NF-kB, PI3K/Akt/mTOR and OPG/RANK/RANKL pathways (43-53). It has been revealed that $\mathrm{mCRP}$ can activate the NF- $\mathrm{\kappa B}$ signaling pathway in mouse and human chondrocytes and induce the expression of proinflammatory cytokines (54). Boras et al (55) determined that mCRP can co-activate PI3K signaling pathway with Notch-3. A previous study demonstrated that mCRP induced osteoclast differentiation by binding to RANKL (56). The aforementioned studies demonstrated that the NF- $\kappa \mathrm{B}$, $\mathrm{PI} 3 \mathrm{~K} / \mathrm{Akt} / \mathrm{mTOR}$ and OPG/RANK/RANKL pathways may be associated with mCRP function.

In conclusion, the present results revealed that there are significant differences in the levels of CRP and $\mathrm{mCRP}$ between patients with $\mathrm{OA}$ and healthy controls, and in different disease stages. Thus, CRP and mCRP may be important serological markers for the early diagnosis and disease evaluation of OA. Additionally, it was also identified in the present study that CRP is significantly correlated with mCRP in OA, which is consistent with previous studies that CRP plays a vital role in diseases through dissociation into mCRP $(18,39)$. However, whether $\mathrm{mCRP}$ is directly involved in the pathogenesis of OA needs further investigation. The present study may have certain limitations, which are reflected in the relatively small sample size and lack of KL1 and KL2 patients. Further expanding the number of patients with KL grades can provide an improved basis and reliability for follow-up research. Whether the combined analysis with other serum markers of OA, such as cartilage oligomeric matrix protein or C-telopeptide fragments of type II collagen, can improve the accuracy of association and prediction remains to be studied in the future. At present, there is no validation of the mCRP in animal models and related mechanism research. In future, using OA animal models can further investigate whether mCRP regulates the pathogenesis of OA. The present study suggested that mCRP may be used as a novel biomarker to help the early diagnosis and severity evaluation of OA and also lays a foundation for in-depth exploration of the pathogenesis of OA, particularly with respect to the role played by $\mathrm{mCRP}$, and future clinical research for therapeutic targets.

\section{Acknowledgements}

The authors would like to thank Professor Yi Wu (Xi'an Jiaotong University) for his constructive advice regarding data analysis and manuscript writing.

\section{Funding}

The present study was supported by the National Natural Science Foundation of China (grant no. 31800654) and the Fundamental Research Funds for the Central Universities (grant no. sxzy012019076).

\section{Availability of data and materials}

The datasets used and/or analyzed during the current study are available from the corresponding author on reasonable request.

\section{Authors' contributions}

HL and PX designed the research. YL, KX and WL performed the experiments. XL and PY analyzed the data and wrote the article. HL, PX and YL confirm the authenticity of all the raw 
data. All authors reviewed the results and read and approved the final version of the manuscript.

\section{Ethics approval and consent to participate}

The present study followed the guidelines of The Declaration of Helsinki and was approved (approval no. 202003055) by the ethics committee of the Hong Hui Hospital (Xi'an, China). Informed consent was obtained from all subjects involved in the present study.

\section{Patient consent for publication}

Not applicable.

\section{Competing interests}

The authors declare that they have no competing interests.

\section{References}

1. Ringdahl E and Pandit S: Treatment of knee osteoarthritis. Am Fam Physician 83: 1287-1292, 2011.

2. Glyn-Jones S, Palmer AJ, Agricola R, Price AJ, Vincent TL, Weinans H and Carr AJ: Osteoarthritis. Lancet 386: 376-387, 2015.

3. Bijlsma JW, Berenbaum F and Lafeber FP: Osteoarthritis: An update with relevance for clinical practice. Lancet 377 : 2115-2126, 2011.

4. Towheed TE, Maxwell L, Anastassiades TP, Shea B, Houpt J, Robinson V, Hochberg MC and Wells G: Glucosamine therapy for treating osteoarthritis. Cochrane Database Syst Rev 2005: CD002946, 2005.

5. Uebelhart D, Malaise M, Marcolongo R, de Vathaire F, Piperno M, Mailleux E, Fioravanti A, Matoso L and Vignon E: Intermittent treatment of knee osteoarthritis with oral chondroitin sulfate: A one-year, randomized, double-blind, multicenter study versus placebo. Osteoarthritis Cartilage 12: 269-276, 2004.

6. Benito MJ, Veale DJ, FitzGerald O, van den Berg WB and Bresnihan B: Synovial tissue inflammation in early and late osteoarthritis. Ann Rheum Dis 64: 1263-1267, 2005.

7. Liu-Bryan R: Synovium and the innate inflammatory network in osteoarthritis progression. Curr Rheumatol Rep 15: 323, 2013.

8. Du Clos TW: Pentraxins: Structure, function, and role in inflammation. ISRN Inflamm 2013: 379040, 2013.

9. Pepys MB and Hirschfield GM: C-reactive protein: A critical update. J Clin Invest 111: 1805-1812, 2003.

10. Gabay C and Kushner I: Acute-phase proteins and other systemic responses to inflammation. N Engl J Med 340: 448-454, 1999.

11. Medzhitov R: Recognition of microorganisms and activation of the immune response. Nature 449: 819-826, 2007.

12. Schwedler SB, Filep JG, Galle J, Wanner C and Potempa LA: C-reactive protein: A family of proteins to regulate cardiovascular function. Am J Kidney Dis 47: 212-222, 2006.

13. Casas JP, Shah T, Hingorani AD, Danesh J and Pepys MB C-reactive protein and coronary heart disease: A critical review. J Intern Med 264: 295-314, 2008.

14. Verma S, Devaraj S and Jialal I: Is C-reactive protein an innocent bystander or proatherogenic culprit? C-reactive protein promotes atherothrombosis. Circulation 113: 2135-2150; discussion 2150, 2006.

15. Bharadwaj D, Stein MP, Volzer M, Mold C and Du Clos TW: The major receptor for C-reactive protein on leukocytes is fcgamma receptor II. J Exp Med 190: 585-590, 1999.

16. Marjon KD, Marnell LL, Mold C and Du Clos TW: Macrophages activated by $\mathrm{C}$-reactive protein through $\mathrm{Fc}$ gamma RI transfer suppression of immune thrombocytopenia. J Immunol 182: 1397-1403, 2009.

17. Li Y,Lee PY, Sobel ES, Narain S, Satoh M, Segal MS, Reeves WH and Richards HB: Increased expression of FcgammaRI/CD64 on circulating monocytes parallels ongoing inflammation and nephritis in lupus. Arthritis Res Ther 11: R6, 2009.
18. Wu Y, Potempa LA, El Kebir D and Filep JG: C-reactive protein and inflammation: Conformational changes affect function. Biol Chem 396: 1181-1197, 2015.

19. Potempa LA, Maldonado BA, Laurent P, Zemel ES and Gewurz H: Antigenic, electrophoretic and binding alterations of human C-reactive protein modified selectively in the absence of calcium. Mol Immunol 20: 1165-1175, 1983.

20. Potempa LA, Yao ZY, Ji SR, Filep JG and Wu Y: Solubilization and purification of recombinant modified $\mathrm{C}$-reactive protein from inclusion bodies using reversible anhydride modification. Biophys Rep 1: 18-33, 2015.

21. Li HY, Wang J, Meng F, Jia ZK, Su Y, Bai QF, Lv LL, Ma FR, Potempa LA, Yan YB, et al: An intrinsically disordered motif mediates diverse actions of monomeric C-reactive protein. J Biol Chem 291: 8795-8804, 2016.

22. Ying SC, Gewurz H, Kinoshita CM, Potempa LA and Siegel JN: Identification and partial characterization of multiple native and neoantigenic epitopes of human C-reactive protein by using monoclonal antibodies. J Immunol 143: 221-228, 1989.

23. Ying SC, Shephard E, de Beer FC, Siegel JN, Harris D, Gewurz BE, Fridkin M and Gewurz H: Localization of sequence-determined neoepitopes and neutrophil digestion fragments of C-reactive protein utilizing monoclonal antibodies and synthetic peptides. Mol Immunol 29: 677-687, 1992.

24. Kohn MD, Sassoon AA and Fernando ND: Classifications in brief: Kellgren-lawrence classification of osteoarthritis. Clin Orthop Relat Res 474: 1886-1893, 2016.

25. Petersson IF, Boegard T, Saxne T, Silman AJ and Svensson B: Radiographic osteoarthritis of the knee classified by the Ahlback and Kellgren \& Lawrence systems for the tibiofemoral joint in people aged 35-54 years with chronic knee pain. Ann Rheum Dis 56: 493-496, 1997.

26. Macri EM, Runhaar J, Damen J, Oei EH and Bierma-Zeinstra SM: Kellgren \& Lawrence grading in cohort studies: Methodological update and implications illustrated using data from the CHECK cohort. Arthritis Care Res (Hoboken). Jan 15, 2021. (Epub ahead of print). doi: 10.1002/acr.24563.

27. Emrani PS, Katz JN, Kessler CL, Reichmann WM, Wright EA, McAlindon TE and Losina E: Joint space narrowing and Kellgren-Lawrence progression in knee osteoarthritis: An analytic literature synthesis. Osteoarthritis Cartilage 16: 873-882, 2008.

28. The R Core Team: R: A language and environment for statistical computing. Reference index. https://cran.r-project.org/doc/ manuals/r-release/fullrefman.pdf. Accessed October 10, 2020.

29. Woolf AD and Pfleger B: Burden of major musculoskeletal conditions. Bull World Health Organ 81: 646-656, 2003.

30. Li HY, Liu XL, Liu YT, Jia ZK, Filep JG, Potempa LA, Ji SR and Wu Y: Matrix sieving-enforced retrograde transcytosis regulates tissue accumulation of C-reactive protein. Cardiovasc Res 115: 440-452, 2019.

31. Pepys MB, Hirschfield GM, Tennent GA, Gallimore JR, Kahan MC, Bellotti V, Hawkins PN, Myers RM, Smith MD, Polara A, et al: Targeting C-reactive protein for the treatment of cardiovascular disease. Nature 440: 1217-1221, 2006.

32. Griselli M, Herbert J, Hutchinson WL, Taylor KM, Sohail M, Krausz T and Pepys MB: C-reactive protein and complement are important mediators of tissue damage in acute myocardial infarction. J Exp Med 190: 1733-1740, 1999.

33. Diehl EE, Haines GK III, Radosevich JA and Potempa LA: Immunohistochemical localization of modified C-reactive protein antigen in normal vascular tissue. Am J Med Sci 319: 79-83, 2000.

34. Schwedler SB, Amann K, Wernicke K, Krebs A, Nauck M, Wanner C, Potempa LA and Galle J: Native C-reactive protein increases whereas modified C-reactive protein reduces atherosclerosis in apolipoprotein E-knockout mice. Circulation 112: 1016-1023, 2005.

35. Ullah N, Ma FR, Han J, Liu XL, Fu Y, Liu YT, Liang YL, Ouyang $\mathrm{H}$ and Li HY: Monomeric C-reactive protein regulates fibronectin mediated monocyte adhesion. Mol Immunol 117: 122-130, 2020.

36. Li QY, Li HY, Fu G, Yu F, Wu Y and Zhao MH: Autoantibodies against $\mathrm{C}$-reactive protein influence complement activation and clinical course in lupus nephritis. J Am Soc Nephrol 28: 3044-3054, 2017.

37. Tan $\mathrm{Y}$, Yu F, Yang $\mathrm{H}$, Chen $\mathrm{M}$, Fang $\mathrm{Q}$ and Zhao $\mathrm{MH}$ : Autoantibodies against monomeric C-reactive protein in sera from patients with lupus nephritis are associated with disease activity and renal tubulointerstitial lesions. Hum Immunol 69: 840-844, 2008. 
38. Yao Z, Zhang $\mathrm{Y}$ and $\mathrm{Wu} \mathrm{H}$ : Regulation of C-reactive protein conformation in inflammation. Inflamm Res 68: 815-823, 2019.

39. Ji SR, Wu Y, Zhu L, Potempa LA, Sheng FL, Lu W and Zhao J: Cell membranes and liposomes dissociate C-reactive protein (CRP) to form a new, biologically active structural intermediate: mCRP(m). FASEB J 21: 284-294, 2007.

40. Ji SR, Wu Y, Potempa LA, Qiu Q and Zhao J: Interactions of C-reactive protein with low-density lipoproteins: Implications for an active role of modified C-reactive protein in atherosclerosis. Int J Biochem Cell Biol 38: 648-661, 2006.

41. Ji SR, Wu Y, Potempa LA, Liang YH and Zhao J: Effect of modified C-reactive protein on complement activation: A possible complement regulatory role of modified or monomeric C-reactive protein in atherosclerotic lesions. Arterioscler Thromb Vasc Biol 26: 935-941, 2006.

42. Khreiss T, Jozsef L, Potempa LA and Filep JG: Loss of pentameric symmetry in C-reactive protein induces interleukin- 8 secretion through peroxynitrite signaling in human neutrophils. Circ Res 97: 690-697, 2005.

43. Fernandez-Torres J, Zamudio-Cuevas Y, Lopez-Reyes A, Garrido-Rodríguez D, Martínez-Flores K, Lozada CA, Muñóz-Valle JF, Oregon-Romero E and Martínez-Nava GA: Gene-gene interactions of the Wnt/ $\beta$-catenin signaling pathway in knee osteoarthritis. Mol Biol Rep 45: 1089-1098, 2018.

44. Wang Y, Fan X, Xing L and Tian F: Wnt signaling: A promising target for osteoarthritis therapy. Cell Commun Signal 17: 97 , 2019.

45. Liu Z, Ren Y, Mirando AJ, Wang C, Zuscik MJ, O'Keefe RJ and Hilton MJ: Notch signaling in postnatal joint chondrocytes, but not subchondral osteoblasts, is required for articular cartilage and joint maintenance. Osteoarthritis Cartilage 24: 740-751, 2016.

46. Liu Z, Chen J, Mirando AJ, Wang C, Zuscik MJ, O'Keefe RJ and Hilton MJ: A dual role for NOTCH signaling in joint cartilage maintenance and osteoarthritis. Sci Signal 8: ra71, 2015.

47. Choi MC, Jo J, Park J, Kang HK and Park Y: NF-kB signaling pathways in osteoarthritic cartilage destruction. Cells 8: 734, 2019.
48. Zhang LB, Man ZT, Li W, Zhang W, Wang XQ and Sun S: Calcitonin protects chondrocytes from lipopolysaccharide-induced apoptosis and inflammatory response through MAPK/Wnt/NF- $\kappa$ B pathways. Mol Immunol 87: 249-257, 2017.

49. Musumeci G, Castrogiovanni P, Trovato FM, Weinberg AM, Al-Wasiyah MK, Alqahtani MH and Mobasheri A: Biomarkers of chondrocyte apoptosis and autophagy in osteoarthritis. Int J Mol Sci 16: 20560-20575, 2015

50. Xue JF, Shi ZM,Zou J and Li XL: Inhibition of PI3K/AKT/mTOR signaling pathway promotes autophagy of articular chondrocytes and attenuates inflammatory response in rats with osteoarthritis. Biomed Pharmacother 89: 1252-1261, 2017.

51. Koura HM, Zaki SM, Ismail NA, Salama EE, El Lebedy DH and Effat LK: Relationship between biochemical bone markers and bone mineral density in patients with phenylketonuria under restricted diet. Iran J Pediatr 24: 23-28, Epub 2013 Dec 31, 2014.

52. Liu Y, Ge J, Chen D, Weng Y, Du H, Sun Y and Zhang Q Osteoprotegerin deficiency leads to deformation of the articular cartilage in femoral head. J Mol Histol 47: 475-483, 2016.

53. Kovacs B, Vajda E and Nagy EE: Regulatory effects and interactions of the Wnt and OPG-RANKL-RANK signaling at the bone-cartilage interface in osteoarthritis. Int J Mol Sci 20: 4653 , 2019.

54. Sproston NR and Ashworth JJ: Role of C-reactive protein at sites of inflammation and infection. Front Immunol 9: 754, 2018.

55. Boras E, Slevin M, Alexander MY, et al: Monomeric C-reactive protein and Notch-3 co-operatively increase angiogenesis through PI3K signalling pathway. Cytokine 69: 165-179, 2014.

56. Jia ZK, Li HY, Liang YL, Potempa LA, Ji SR and Wu Y. Monomeric C-reactive protein binds and neutralizes receptor activator of NF- $\mathrm{BB}$ ligand-induced osteoclast differentiation. Front Immunol 9: 234, 2018.

This work is licensed under a Creative Commons Attribution-NonCommercial-NoDerivatives 4.0 International (CC BY-NC-ND 4.0) License. 